\title{
La dynamique éthique : une approche participative pour améliorer les conditions de travail
}

\author{
Marc Jean \\ Université du Québec à Chicoutimi, \\ Jacqueline Dionne-Proulx \\ Télé-Université (UQAM)
}

\section{Introduction}

« Dans le contexte économique et social actuel, parler d'éthique de l'organisation nous paraît une démarche non seulement ambitieuse sur le plan collectif, mais combien utile pour l'individu qui cherche à construire sa vie parfois désespérément pour et par les organisations. En effet, l'individu, souvent ballotté dans le mouvement incessant de création et de destruction des organisations, fait face alors à un grand vide » disent Sautré et Ganier (2007) ${ }^{1}$.

L'objectif de cet article est de montrer qu'il est possible de réintroduire la personne-sujet au centre de l'organisation grâce à la dynamique éthique. Cette démarche nous amène à repositionner l'éthique comme mode de réflexion et d'action au cour des préoccupations de l'organisation. En quoi et comment est-ce possible? C'est possible en ce que tout membre d'une organisation et à quelque niveau de hiérarchie qu'il se trouve cherche le sens à donner à sa pratique au quotidien et s'attend de combler l'écart qui sépare sa pratique de l'idéal professionnel visé. C'est possible en mettant de l'avant l'intégration d'une dynamique éthique de manière à ce que chaque participant à cette vie organisationnelle soit placé et se reconnaisse comme partie prenante et participe à ce projet de vie.

\section{Une dynamique éthique, un milieu de vie, un questionnement}

La dynamique éthique se caractérise par une réflexion critique qui se situe en aval et en amont des normes, des principes d'action et de toute forme de pratiques qui guide l'action humaine dans la recherche d'un mieux vivre ensemble. Ricoeur $(1990)^{2}$ note d'ailleurs que l'éthique en situation de travail, c'est l'action qui consiste à construire et à améliorer son rapport à soi (acteur apprécie son action dans une quête d'estime de soi), aux autres (il se préoccupe de l'effet de sa conduite sur les autres) et à l'entreprise (mise en concordance de son projet personnel en rapport avec celui de l'entreprise en vue de dégager du sens à l'action), dans une perspective de développement personnel et collectif. Une dynamique éthique permet donc une cohérence entre les niveaux stratégique et opérationnel et la recherche de sens partagé. De même, elle émerge moins d'une perspective d'hétéronomie avec un ensemble de règles fixes que d'un processus continu de découverte et de compréhension des principes sous-jacents qui guident les actions, et ce à travers une rencontre avec d'autres personnes. En ce sens, la dynamique éthique conduit l'individu à redevenir sujet (figure 1). 
Figure 1 : La dynamique éthique au sein de l'organisation

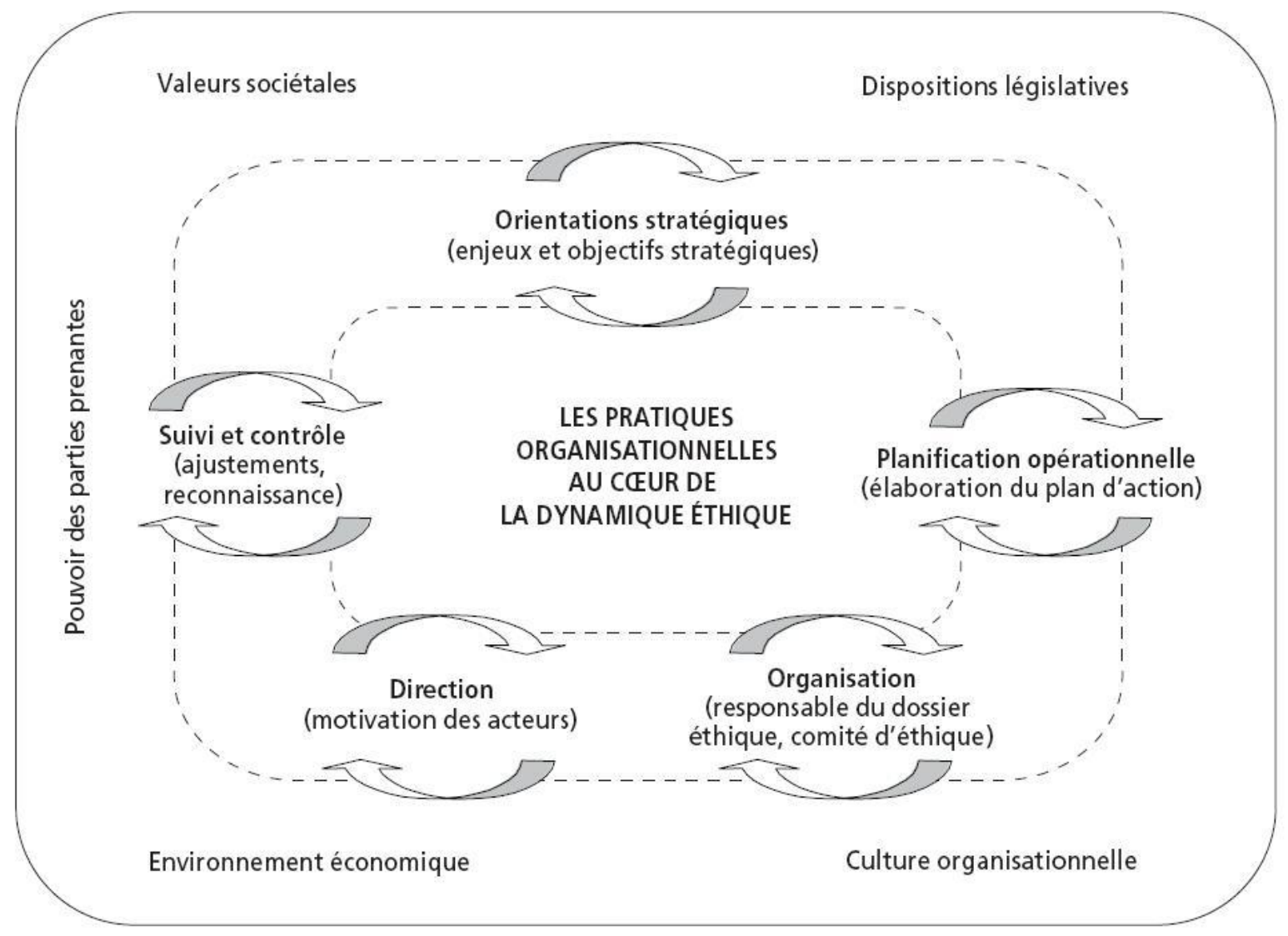

Les pratiques opérationnelles sont centrales dans la dynamique éthique. Elles constituent tantôt des objets qui permettent d'interroger et de faire interagir de l'intérieur les trois grandes préoccupations de toute organisation, soit les objectifs poursuivis, le bien fondé de ces objectifs et les résultats en liant les plans stratégiques et les pratiques opérationnelles de façon à assurer le déroulement harmonieux des processus opérationnels. Tantôt elles réfèrent à des sujets qui quotidiennement tentent de donner un sens à ces pratiques, et donc à leurs actions, à travers des valeurs et des principes qui les colorent et des problématiques qui les structurent.

La démarche de gestion favorise le déploiement de cette dynamique dans l'organisation. Elle s'intéresse à l'encadrement de l'équipe, sa motivation, ses résultats, admet la nécessité de politiques, procédures, normes. Par ailleurs, une série de boucles itératives permet d'assurer les ajustements requis de telle sorte que les objectifs stratégiques en viennent à donner de la cohérence aux pratiques organisationnelles et que les acteurs, dans leurs pratiques, puissent y trouver du sens.

La prise en compte des dimensions souvent macro-éthiques (contexte sociétal, environnement organisationnel, etc.) enrichit la dynamique éthique et permet que se développe un niveau de préoccupations empreint d'objectivité propre à alimenter le «soi » des acteurs de manière à faire en sorte que ce soit également de l'intérieur que la vie organisationnelle se trouve régénérée. Elle marque enfin la prise en compte de l'environnement dans lequel l'organisation œuvre. 
Il s'agit donc d'un habile compromis qui permet à la fois de tenir compte des normativités, des politiques et procédures, autrement dit des modes de régulation existants dans les organisations, et d'une régulation autonome fondée sur un échange constructif en présence d'une collectivité donnée. L'hétéronomie et l'autonomie

\section{Une dynamique éthique, trois démarches}

Même s'il est attendu, voire souhaité, que toute démarche initiée en milieu organisationnel pour que se déploie le sens que les acteurs donneront individuellement et collectivement à leurs pratiques s'inscrive dans une perspective éthique, c'est à différents niveaux que la contribution de tous et chacun sera sollicitée. Les trois démarches proposées dans le cadre de la dynamique éthique ont été largement documentées par Dionne-Proulx \& Jean (2007) dans Pour une dynamique éthique au sein de l'organisation'.

\subsection{La démarche organisationnelle à caractère éthique}

Habituellement fondée sur des droits et obligations définies dans des lois et règlements, tels l'équité, la nondiscrimination, etc., certains principes ou certaines règles dans les organisations permettent d'établir des façons de faire qui favorisent un vivre-ensemble harmonieux. Lorsque, dans une perspective d'une plus grande harmonisation avec le milieu, l'organisation cherche à remettre en cause ou simplement questionner ces normativités, nous disons alors que se déclenche une démarche organisationnelle à caractère éthique. Dans ce cadre, la motivation qui pousse les acteurs à se conformer aux politiques et aux normes établies vient habituellement de la reconnaissance de l'autorité de la personne qui a énoncé cette norme et de la légitimité de celle-ci. peuvent ainsi cohabiter au sein de l'entreprise dans une dynamique qui veut réintroduire la personne-sujet au centre de l'organisation.

Quelles sont donc les démarches propres à susciter une telle dynamique éthique? Voici comment nous entrevoyons répondre ici à cette interrogation.

La principale critique de cette approche serait que l'on appelle « éthique » des pratiques qui visent en fait des règles de conduite assimilables à un règlement interne de l'organisation. Dans la mesure où la démarche débouche sur des normes édictées dans un but de régulation des conduites, sans discussion, alors elle risque d'entrer fondamentalement en contradiction avec l'exigence éthique de liberté et de responsabilisation des acteurs. Dès lors, elle suppose que l'on mise sur l'obéissance hiérarchique et sur le contrôle pour en assurer la réalisation puisque rien ne garantit que les acteurs intériorisent les règles et les principes édictés.

Dans le cadre de la mouvance des transformations économiques et technologiques, il apparaît indispensable de responsabiliser les acteurs organisationnels afin de remettre en permanence sous tension les institutions, les pratiques et les règles existantes. Aussi est-il important d'introduire dans le milieu une autre démarche complémentaire à celle-ci, soit la démarche éthique axée sur les valeurs.

\subsection{La démarche éthique axée sur les valeurs}

Dans une gestion renouvelée, quelle place y at-il lieu de faire à l'autonomie et à la responsabilisation des personnes? Même si celle-ci est au cœur du débat depuis quelque temps dans le monde de la gestion, les discussions n'ont pas permis de trancher sur la 
manière de concevoir une véritable autonomie individuelle au sein de l'entreprise.

L'autonomie est alors moins une réalité qu'un projet à définir au sein de l'entreprise collectif pour que les orientations à donner à la mission et aux pratiques deviennent le
Dans le cadre de la démarche éthique axée sur les valeurs, la personne se trouve recentrée comme sujet et objet de son expérience et de ses pratiques organisationnelles, donc apte à faire un usage éclairé de son autonomie, au fur et à mesure qu'elle prend forme. L'autonomie est alors moins une réalité qu'un projet à définir au sein de l'entreprise. Elle s'exerce à travers un processus réflexif et un dialogue et conduit à une prise de décision éclairée. Elle appelle à la créativité et au sens novateur des acteurs organisationnels dans le cadre d'une réflexion sur les valeurs.

Dès lors, le collectif prend ses décisions et agit ultimement de manière congruente avec les valeurs dont la signification s'accroît lorsqu'elles sont partagées par l'ensemble des personnes qui forment le groupe. Les valeurs deviennent alors un repère pour chacun des travailleurs appelé à mesurer les conséquences de ses décisions et de ses actions sur soi, les autres et l'organisation. Petit à petit, c'est tout un processus de responsabilisation que les personnes réalisent et ce processus leur permet de développer la conviction que c'est à travers le souci des autres que le soi se constitue. En somme, la démarche amène les individus à prendre des décisions considérées par tous comme étant raisonnables ou justes et donc des décisions qui sont les meilleures, compte tenu des circonstances dans lesquelles elles se prennent.

La démarche éthique axée sur les valeurs peut se comprendre comme un effort individuel et reflet des personnes qui les habitent, et ce, au nom de la dignité humaine. C'est alors qu'une éthique organisationnelle peut prendre la forme d'un fil conducteur qui rejoint toute actrice ou acteur de manière à ce qu'ils deviennent dans la même perspective, porteurs des valeurs de la collectivité et de la mission que l'organisation cherche à refléter à l'intérieur de la société toujours pour un mieux-être et un mieux vivre ensemble.

\subsection{La démarche déontologique}

La démarche déontologique renvoie à un ensemble de responsabilités, devoirs et obligations dont les individus et les collectivités professionnelles sont porteurs et qu'ils sont susceptibles de reconduire dans des codes, des chartes ou des guides de conduite en vue d'une meilleure régulation de ces dernières dans une perspective de plus grande cohérence des personnes et d'une plus grande cohésion sociale. Quant à la démarche déontologique à plus proprement parler, nous croyons que la mise en place d'un tel processus nécessite, comme pour la démarche éthique axée sur les valeurs, de faire appel aux sources vives du milieu organisationnel pour initier un code de déontologie ou un code d'éthique, un guide des conduites professionnelles, etc. Mission, principes, valeurs, tels sont des exemples de composantes à mettre à contribution pour la mise en place de tels outils.

\section{Les défis de l'intégration d'une dynamique éthique dans les organisations}

La volonté d'intégrer une dynamique éthique aux pratiques organisationnelles par le biais d'une démarche déontologique, tout comme une démarche organisationnelle à caractère éthique et une démarche éthique axée sur les valeurs représente un certain nombre de défis 
pour les différents acteurs organisationnels et appelle un arrimage entre les valeurs personnelles et professionnelles des acteurs et les valeurs organisationnelles.

\subsection{Faire cohabiter hétéronomie et autonomie au sein de l'entreprise}

Comme nous l'avons mentionné précédemment, pour que s'actualise une dynamique éthique, il est nécessaire que celleci repose sur un système de gestion qui allie normativités et responsabilisation des personnes afin de réintroduire la personnesujet au centre de l'organisation. Cela veut dire qu'il est essentiel que l'entreprise puisse être en mesure de donner aux acteurs organisationnels les moyens de s'interpréter dans un horizon de sens et selon des valeurs incarnées et des normes et des politiques qui font consensus le plus possible. Cette cohabitation hétéronomie-autonomie suppose en réalité une interaction dynamique entre les différents niveaux stratégique et opérationnel et les différentes activités au sein de l'organisation. Elle implique une démocratisation des rapports d'autorité, une responsabilisation accrue des travailleurs, une autonomie plus grande et une coopération plus développée dans l'entreprise. L'association des partenaires organisationnels comme coconstructeurs dans le processus de définition des valeurs organisationnelles permet de prendre en compte une pluralité de valeurs.

De plus, l'autonomie et la responsabilisation des acteurs doivent s'exercer de manière responsable et compatible avec les objectifs d'efficacité et de performance de l'entreprise (résultats). Pour y arriver, les initiatives ne doivent pas rester à la périphérie des structures de décisions ou en parallèle avec les paliers organisationnels. Elles doivent s'imbriquer au sein de l'organisation réelle du travail. C'est donc à une démarche concertée qui suppose des changements au plan des valeurs, des attitudes et des comportements que les parties sont conviées. Il pourrait s'agir là d'une importante contribution à l'émergence d'une nouvelle culture organisationnelle misant sur l'intelligence créatrice des travailleurs.

\subsection{Favoriser le déploiement de valeurs partagées}

Un deuxième grand défi pour les entreprises réside dans le fait de favoriser le déploiement de valeurs partagées afin que les normes établies, les décisions prises aient du sens aux yeux des acteurs et fassent ultimement consensus.

Notre perspective consiste à reconnaître que c'est dans le dialogue au sein des équipes de travail que se circonscrivent les valeurs desquelles s'inspirent plus ou moins consciemment les acteurs comme individus et comme collectivités. Ces valeurs partagées par la base peuvent ensuite contribuer au déploiement de la charte de valeurs qui devient alors un repère de premier plan pour guider les actions dans l'organisation.

De plus, pour que ces valeurs soient réactualisées régulièrement, que les nouveaux venus les intériorisent, celles-ci doivent faire l'objet d'un dialogue continu. Ce dialogue est nécessaire, comme le mentionnent Boisvert et al. $(2003)^{4}$, puisque l'éthique apparaît en fonction de la différence (valeurs, intérêts) entre soi et l'autre, le rapport à l'autre passant par la reconnaissance de celui-ci en tant que personne. Dès lors, le respect de son identité se traduit par la prise en compte de ses intérêts dans l'évaluation des décisions et actions. Cette exigence éthique vise à résoudre la tension entre autonomie et respect de l'autre, favorisant ainsi un mieux-vivre ensemble.

3.3 Assurer la construction de la dynamique éthique par la transformation sociale de l'organisation et des acteurs de l'organisation

Tout comme il est essentiel que les acteurs 
organisationnels acquièrent des connaissances et des aptitudes pour mener à bien leurs tâches, ils doivent également acquérir des connaissances de base et développer des habiletés pour prendre des décisions éclairées et adopter des pratiques qui concilient l'éthique et les réalités économiques de l'entreprise.

Ce qui est recherché, c'est une transformation des processus d'actions et d'interactions au niveau des personnes et des groupes de façon à assurer que les changements opérés permettent l'intégration d'un sens éthique, élaboré librement et collectivement. La démarche d'intervention participative est privilégiée ici parce qu'elle favorise une approche collective, réflexive et qu'elle permet de situer l'acteur social dans la problématique de l'élaboration $\mathrm{du}$ sens en contexte, et, particulièrement de l'impliquer au sein de ce contexte (approche participative) tout en l'accompagnant de façon à ce qu'il en vienne, avec le temps, à disposer de ses propres ressources pour maintenir ou engendrer les changements requis.

À titre d'exemple, la démarche d'intervention participative peut inclure des activités tels la pratique du dialogue et les cercles de santé. Ces derniers, mis en place en Allemagne, constituent un bel exemple de projets participatifs qui ont donné des résultats. Ces cercles ont démontré leur efficacité dans un grand nombre d'entreprises (11 études dans 30 compagnies différentes) avec des effets positifs sur la santé, sur la satisfaction et la motivation, sur le processus organisationnel avec plus d'efficience au niveau de la charge de travail et de la communication (European Agency for Safety and Health at Work, 2008) ${ }^{5}$. Quant à la pratique du dialogue, lorsque celleci est intégrée dans les routines quotidiennes d'une organisation, les personnes changent de façon permanente leurs manières de penser et d'agir. La pratique du dialogue a aussi été décrite comme un des éléments majeurs de «l'apprentissage organisationnel» permettant aux entreprises de devenir efficientes dans le sens où les changements opérés perdurent.

D'autres activités peuvent également favoriser la prise en charge. L'entreprise peut d'abord miser sur des ateliers de sensibilisation et d'accompagnement. Au cours de ces ateliers, des thèmes généraux comme la communication, l'autonomie, la prise de décision et des thèmes plus spécifiques tels les valeurs, le conflit de valeurs, le dilemme et sa résolution, les finalités et la légitimité des moyens peuvent être abordés. Le but de ces ateliers est d'apporter des clarifications, des éléments de réflexion afin d'aider les participants à mieux comprendre les différents concepts entourant le déploiement d'une dynamique éthique et surtout à en découvrir les applications possibles dans leurs lieux de travail. Aussi, dans le but d'assurer le transfert des acquis dans le milieu de travail, les acteurs organisationnels peuvent être invités lors de ces ateliers à venir témoigner des pratiques qui leur permettent d'incarner les valeurs qu'ils partagent et analyser de façon interactive l'ensemble de leurs pratiques quotidiennes à la lumière de ces pratiques. Il s'agit de promouvoir les pratiques qui respectent l'esprit des valeurs énoncées et des normes en place et de laisser tomber celles qui s'en écartent.

À cela peuvent s'ajouter des ateliers visant le développement de la réflexion critique ou encore la mise en place d'outils d'apprentissage interactifs ou non, à partir de dilemmes rencontrés en situation de travail. Des exposés et des apports de spécialistes en éthique tout au long de cette discussion permettent de proposer des repères plutôt que des réponses en vue de renvoyer chacun aux valeurs partagées.

Une telle trousse d'outils est encore à développer et suppose une pédagogie dynamique qui propose de passer d'un exposé didactique à un travail de discussion en petits 
groupes. Mais elle est susceptible de mobiliser les travailleurs, de leur permettre de trouver en équipe des solutions créatrices aux défis éthiques qui sont les leurs. De même, il est possible de développer des outils interactifs susceptibles de susciter la réflexion individuelle et collective qui pourraient être réellement utilisés pour un grand nombre d'entreprises afin de permettre aux travailleurs d'évoluer avec leur emploi et de s'adapter aux divers changements du milieu de travail. Tout un chantier est ici ouvert.

\section{Il est possible de développer des outils interactifs susceptibles de susciter la réflexion individuelle et collective}

Nous partageons également le constat de certains auteurs qui sont d'avis que la mobilisation des travailleurs est possible dans la mesure où ceux-ci peuvent faire l'exercice concret vers une plus grande autonomie. Ainsi, il est possible de permettre aux travailleurs d'exercer un droit d'opposition et un droit d'initiative lorsque des ordres hiérarchiques sont susceptibles de mettre en jeu la responsabilité pénale ou disciplinaire de celui qui agit, tout comme il est possible au Québec pour un travailleur de refuser d'exécuter un travail dangereux en vertu de la Loi sur la santé et la sécurité du travail. Un autre moyen concret d'assurer, dans l'entreprise, la primauté des valeurs inscrites au code de valeurs partagées serait de permettre à tout membre d'une équipe qui s'est définie des valeurs, de poser des questions, de susciter un débat s'il considère qu'il y a un écart entre la situation qu'il vit et une ou des valeurs partagées par l'équipe. Ces droits sont des moyens concrets pour permettre aux différents acteurs organisationnels d'exercer leur responsabilité morale dans le cadre de leurs occupations.
Il s'avère nécessaire sinon indispensable que la direction crée les conditions favorables pour que le collectif puisse se prendre en main, c'est-à-dire que les acteurs de l'entreprise puissent partager le sentiment que la dynamique éthique est légitime.

\subsection{S'assurer d'une cohérence dans la structure et le fonctionnement des organisations}

Enfin, un dernier défi consiste à traduire cette dynamique éthique, dont les valeurs sont coconstruites, dans les politiques écrites et le vécu quotidien. Il s'agit de se doter de structures qui permettent d'opérationnaliser les repères éthiques dans la gestion de tous les jours en tenant compte des nombreuses normativités existantes dans les entreprises. En fait, cela suppose de travailler sur les liens entre les valeurs, les politiques et les procédures, les principes managériaux et les situations de travail au quotidien. En ce sens, les gestionnaires, les travailleurs sont conviés à s'interroger sur leurs décisions et actions : Pourquoi ? Dans quels buts? Avec quelle intention? Ces questionnements permettent de créer de la cohérence entre les discours et l'action.

De même, les structures de rémunération, les systèmes d'évaluation de la performance et la répartition de la prise de décision dans l'entreprise constituent des éléments susceptibles d'influencer les comportements éthiques dans l'entreprise. Ces politiques doivent non seulement être opérantes mais elles doivent être bien appliquées dans l'organisation. Aussi, est-il important, si l'on choisit de récompenser les comportements éthiques, de s'assurer que ces récompenses n'engendreront pas d'effets pervers, soit la recherche de la récompense par tous les moyens possibles...y compris les moyens illicites. 


\section{Conclusion}

En définitive, la dynamique éthique proposée ici s'inscrit dans l'optique d'une plus grande autonomie de l'individu, en tant qu'acteur faisant partie intégrante d'une organisation et ayant pour résultat l'appropriation par celui-ci d'une liberté encore plus certaine. Lorsque l'entreprise s'engage dans un mouvement si fortement porteur de sens, se pose alors la question de la gestion de cet engagement et de son pilotage.

L'expérimentation d'outils novateurs de management susceptibles de développer des activités qui favorisent un renouvellement des valeurs dans l'entreprise s'inscrit alors comme une nécessité dans cette perspective. Réinventer de nouvelles formes d'organisation $\mathrm{du}$ travail qui puissent cohabiter avec l'aspiration des travailleurs à se prendre en charge constitue sans nul doute l'un des défis majeurs du $21^{\text {ième }}$ siècle qui attend les spécialistes de la gestion.

Par ailleurs, point n'est besoin de préciser que le déploiement d'une dynamique éthique quelle que soit la démarche initiée, constitue avant tout une quête de sens et de cohérence de l'agir, une ouverture sur un idéal à atteindre dans l'entreprise dans une perspective de mieux-être et de mieux-vivre ensemble. Par la réflexion et le dialogue, il s'agit enfin d'une invitation à la mise en place d'une démarche d'autonomisation et de responsabilisation encore plus grande de la part de tous les acteurs organisationnels.

\section{Notes et references}

1 Sautré, G., Ganier, P. (2007). «Préface » dans Dionne-Proulx, J., Jean, M., Pour une dynamique éthique au sein de l'organisation, Éditions Télé-université, Ste-Foy : Presses de l’Université du Québec.

2 Ricœur, P. (1990). Soi-même comme un autre, Paris : Éditions Seuil.

3 Dionne-Proulx, J., Jean, M. (2007). Pour une dynamique éthique au sein de l'organisation. Éditions Téléuniversité, Ste-Foy : Presses de l'Université du Québec. Boisvert, Y., Jutras, M., Legault, G.A, Marchildon, A. (2003). Petit manuel d'éthique appliquée à la fonction publique, Éditions Liber, Montréal.

European Agency for Safety and Health at Work. (2002). « Health Circles - A Participative Approach to Improve Health Related Working Conditions - Germany ». In How Tackle Psychosocial Issues and Reduce Work-related Stress. Section 3.3. Report 309 : OSH Systems and Programmes, 40-45. consulté le 18-06-2008. http://osha.europa.eu /publications/reports/309/ 